\title{
Procesamiento de Arvejas (Pisum sativum L.). Parte 4: Optimización del Proceso de Escaldado de Arvejas (Pisum sativum L.) Variedades Sureña y Andina en base a la Inactivación de Peroxidasa
}

\author{
Yamid A. Pinchao, Johana C. Andrade y Oswaldo Osorio* \\ Universidad de Nariño, Facultad de Ingeniería Agroindustrial, Departamento procesos industriales, Grupo \\ de Investigación GAIDA Ciudad Universitaria Torobajo, Calle $18 \mathrm{Cr}$ 50, San Juan de Pasto - Colombia \\ (e-mail: yamidalexis@hotmail.com; joha.intel0705@hotmail.com; osorio_oswaldo@hotmail.com)
}

${ }^{*}$ Autor a quien debe ser dirigida la correspondencia

Recibido Sep. 15, 2015; Aceptado Nov. 6, 2015; Versión final Feb. 5, 2016, Publicado Ago. 2016

\section{Resumen}

Se optimizó el proceso de escaldado de arveja (Pisum sativum L.), variedades sureña y andina, en base a la actividad de la enzima peroxidasa (POD), y se evaluó su efecto sobre el contenido de clorofila total, la textura y el color. Se aplicó un diseño central compuesto $2^{\mathrm{k}}$ con 3 puntos centrales y 2 axiales, con temperatura y tiempo como variables de proceso a 3 niveles, cada una sobre 4 variables respuesta. La cuantificación POD y de clorofila se realizó antes y después del escaldado a través de espectrofotometría UV-VIS a 470, 645 y $663 \mathrm{~nm}$ respectivamente. La textura se determinó por compresión, el color se determinó por medio de coordenadas rectangulares $\left(L^{*}, a^{*}, b^{*}\right)$. El proceso de escaldado optimizado fue de $76.98{ }^{\circ} \mathrm{C}$ por $2.56 \mathrm{~min}$ y de $82.32{ }^{\circ} \mathrm{C}$ por 2.58 min para arveja andina y sureña respectivamente, logrando mantener una actividad residual POD $\leq 10 \%$.

Palabras clave: escaldado; peroxidasa; color; textura; clorofila; Pisum sativum L.

\section{Processing Peas (Pisum sativum L.).Part 4: Blanching Process Optimization Pea (Pisum sativum L.) Cultivares Sureña and Andina based on the Inactivation of Peroxidase}

\begin{abstract}
The blanching process was optimized on base to the activity of peroxidase (POD), and its effect on total chlorophyll content, texture and color in peas (Pisum sativum $L$ ), of the varieties sureña and andina, was evaluated. A $2^{k}$ central mixed design was applied with 3 central and 2 axial points with temperature and time as process variables at 3 levels each and 4 response variables. POD quantification and chlorophyll was performed before and after scalding by UV-VIS spectrophotometry at 645 and 470 and $663 \mathrm{~nm}$, respectively. Texture was determined by compression and color was determined by means of rectangular coordinates $\left(\mathrm{L}^{*}, \mathrm{a}^{*}, \mathrm{~b}^{*}\right)$. The optimum blanching process was $76.98{ }^{\circ} \mathrm{C}$ in $2.56 \mathrm{~min}$ and $82.32{ }^{\circ} \mathrm{C}$ in 2.58 min for andina and sureña pea respectively, achieving constant residual $P O D \leq 10 \%$.
\end{abstract}

Keywords: blanching; peroxidase; color; texture; chlorophyll; Pisum sativum L. 


\section{INTRODUCCION}

El cultivo de arveja en Colombia se concentra en los departamentos de Nariño, Boyacá y Cundinamarca. Nariño se ha convertido en el principal productor nacional, pasando de producir 11.000 toneladas en 2010 a 30.000 toneladas en 2013 y ha mantenido su tendencia de aumento; mientras que los otros dos departamentos solo se mantienen en una producción alrededor de las 8.000 toneladas entre 2010 y 2013 (Agronet, 2013). No obstante, toda la producción Nariñense se comercializa en fresco y por tal razón, se hace necesario evaluar tratamientos térmicos como el escaldado, el cual, permitirá aumentar las formas de industrialización y comercialización de esta leguminosa, debido a que este proceso térmico se utiliza como etapa previa a los procesos de congelación, secado y esterilización. El escaldado tiene como principal objetivo inactivar enzimas, resaltar el color y ablandar el producto para favorecer su posterior envasado y/o almacenamiento (Mendoza y Herrera, 2012; Gökmen et al., 2005).

El escaldado, busca principalmente la destrucción de enzimas que afectan color, sabor y contenido vitamínico de frutas y hortalizas antes de ser congelados, deshidratados o enlatados (Lin y Brewer, 2005; Mazzeo et al., 2015) de ahí que para optimizar este proceso se utilizan enzimas indicadoras, las cuales, sirven para comprobar la eficiencia del proceso, ya que al ser inactivadas durante el tratamiento térmico demuestran que éste se ha realizado de forma eficiente (Anthon y Barrett, 2002; Gökmen et al., 2005). La enzima peroxidasa (POD) es la más utilizada como indicadora del proceso de escaldado, debido principalmente a su alta resistencia térmica y a su facilidad para ser cuantificada (Anthon y Barrett, 2002; Polata et al., 2009). Para evaluar la presencia de peroxidasa, el sustrato comúnmente utilizado es el guayacol, que es oxidado a un complejo coloreado de tetraguayacol de color rojo ladrillo que es usado como medida de la actividad enzimática por lectura de la absorbancia en el espectrofotómetro con respecto al tiempo de reacción de la enzima-sustrato (Anthon y Barrett, 2002; Mazzeo et al., 2015).

Otro de los efectos del escaldado es la remoción de aire atrapado dentro y entre las células vegetales, facilitando alcanzar el vacío requerido en el envase durante procesos de enlatado o conserva (Featherstone, 2015). Por otro lado previene la oxidación de compuestos durante el almacenamiento de productos congelados, ayuda en parte a reducir la contaminación por microorganismos y mejora el color en el producto, aunque en algunos casos se obtiene una disminución del mismo (Petzold et al., 2014). Industrialmente, la técnica de escaldado más comúnmente usada es la de inmersión del alimento en agua a temperaturas entre 70 a $100{ }^{\circ} \mathrm{C}$. El costo de este tipo de instalación es relativamente bajo, y se considera que la transferencia de calor es mejor (Aguilar et al., 1999). Dentro de los parámetros a tener en cuenta durante el proceso de escaldado está la textura que es un atributo importante de calidad que influye en la preferencia del consumidor y puede tomarse como índice de deterioro durante el procesamiento y manipulación de alimentos, ya que sufren cambios durante la aplicación de tratamientos térmicos debido principalmente a gelatinización de almidones y solubilización de sustancias pécticas, provocando la pérdida de firmeza en el tejido vegetal (Mendoza y Herrera, 2012).

Otro de los parámetros es la clorofila, este pigmento (común a todas las células fotosintéticas) es clave para la arveja, ya que durante el proceso de escaldo le otorga el grado de verdor que está en función de la concentración presente de clorofila (Macías et al., 2007; Turkmen et al., 2006). Finalmente cuando los alimentos se someten a tratamientos térmicos, estos desarrollan diferentes tonalidades de color debido a cambios químicos inducidos por las diferentes condiciones de procesamiento, dentro de las cuales están principalmente las altas temperaturas (Icier et al., 2006; Padrón et al., 2012). Estos cambios de color se pueden medir aprovechando la propiedad de cada alimento de reflejar o de absorber una cierta longitud de onda en el espectro visible (Torres et al., 2013). Tomando en cuenta todo lo anterior, los objetivos de este trabajo fueron optimizar el proceso de escaldado en arveja sureña y andina basado en la inactivación de la enzima peroxidasa y evaluar el impacto del tratamiento térmico sobre algunas propiedades fisicoquímicas de las dos variedades de arveja.

\section{MATERIALES Y MÉTODOS:}

Se describe la ubicación donde se realizadron los experimentos, como se onbtuvo la muestra,se explica el tratamiento térmico, luego la extracción de la enzima peroxidasa y la cuantificación de la actividad enzimática. Finalmente se explica la evaluación de las propiedades fisicoquímicas, el diseño experimental y el análisis de resultados

\section{Ubicación}

La investigación se realizó en el Laboratorio de Investigación en Conservación y Calidad de Alimentos de la Universidad de Nariño sede Torobajo, Pasto, Nariño, localizado a 2527 msnm., con una temperatura promedio de $14{ }^{\circ} \mathrm{C}$ y una humedad relativa de $70 \%$. 


\section{Obtención de la muestra}

Se utilizó arveja (Pisum sativum, L) de las variedades sureña y obonuco andina recolectadas en la granja del Centro Internacional de Producción Limpia LOPE, Sena Regional Nariño, ubicado a 2650 msnm con una temperatura promedio de $13 \stackrel{\circ}{\circ}$ y una precipitación promedio anual de $700 \mathrm{~mm}$.

\section{Tratamiento térmico}

El proceso de escaldado se realizó por inmersión en agua caliente acorde a la temperatura y tiempo expresados en el diseño experimental que se muestra más adelante. Se utilizó un baño termostático Eyela OSB 2000 de 10 litros de capacidad, y precisión de temperatura de $\pm 0,1^{\circ} \mathrm{C}$. La temperatura se monitoreó con una termocupla tipo $\mathrm{K}$ acoplada a un lector de temperatura portátil (TECPEL DTM-305). Una vez escaldadas, las muestras fueron transferidas inmediatamente a un baño de agua-hielo a temperatura $\leq 4{ }^{\circ} \mathrm{C}$ con el fin de someterlas a choque térmico (Latorre et al., 2013).

\section{Extracción de la enzima Peroxidasa (POD)}

Se realizó siguiendo la metodología reportada por Latorre et al. (2013). Se maceraron $5 \mathrm{gr}$ de arveja en una cápsula de porcelana y se agregaron $20 \mathrm{~mL}$ de Buffer fosfato de sodio $0,1 \mathrm{M}$ de $\mathrm{pH}=7$; posteriormente, se agitó por 5 min en una plancha de agitación magnética, finalmente se centrifugó la muestra a 10000 rpm durante 15 min a $10^{\circ} \mathrm{C}$ y se usó el sobrenadante para realizar el análisis enzimático.

\section{Cuantificación de la actividad enzimática $P O D$}

Se realizó aplicando la metodología reportada por Latorre et al. (2013). Se utilizó un espectrofotómetro UVVIS (THERMO SCIENTIFIC GENESYS 10UV-VIS). Se preparó una solución de $50 \mathrm{~mL}$ de buffer fosfato de sodio $0,1 \mathrm{M}$ de $\mathrm{pH}=7$, MERCK, $50 \mu \mathrm{L}$ de Guayacol al $99 \%$, MERCK y $50 \mu \mathrm{L}$ de $\mathrm{H}_{2} \mathrm{O}_{2}$ al $30 \%$, MERCK. De esta solución se tomó $3 \mathrm{~mL}$ y se mezcló con $10 \mu \mathrm{L}$ de extracto enzimático, esta reacción formó un complejo coloreado de tetraguayacol (rojo ladrillo) que fue monitoreado a una longitud de onda de $470 \mathrm{~nm}$ cada $30 \mathrm{~s}$ durante los primeros $7 \mathrm{~min}$, tiempo en el cual la reacción responde a una ecuación lineal, $A=m t+b$, donde: $\mathrm{A}$, absorbancia; $\mathrm{m}$, velocidad enzimática $(\Delta \mathrm{A} / \mathrm{min})$; $\mathrm{t}$, tiempo (min) y b, el intercepto (Latorre et al., 2013). De la ecuación lineal se tomó el valor de la pendiente $\mathrm{m}$ como el valor de la actividad enzimática POD (Latorre et al., 2013; Pinchao et al., 2014). Cada análisis de actividad POD se realizó por triplicado a $18{ }^{\circ} \mathrm{C}$. La actividad enzimática residual (AR), se calculó de la relación de velocidad de formación del complejo de tetraguayacol de la enzima después del tratamiento y la velocidad de formación del complejo de tetraguayacol de la enzima sin tratamiento. Su cálculo se realizó utilizando la ecuación (1), reportada por (Tiwari et al., 2009; Latorre et al., 2013; Pinchao et al., 2014) en términos de porcentaje:

$\% A R=\frac{A_{t}}{A_{0}} \times 100$

Donde $A_{t}=$ Actividad enzimática POD después del tratamiento térmico y $A_{0}=$ Actividad enzimática POD sin tratamiento térmico

\section{Evaluación de las propiedades fisicoquímicas}

Textura: Se evaluó por método instrumental reportado por Huerta et al. (2008). Las muestras fueron estudiadas utilizando un analizador de textura LS1 (Test and Calibration Instruments Ametek, Lloyd Materials testing, USA) con vástago cilíndrico de $3 \mathrm{~mm}$ de diámetro, distancia de recorrido de $5 \mathrm{~mm}$ y una velocidad de $5 \mathrm{~mm} / \mathrm{s}$ con regreso automático (Huerta et al., 2008).

Color: Se retiró el exceso de humedad externa de las muestras y se midieron los valores $L^{*}$ (luminosidad), $\mathrm{a}^{*}$ (rojizo-verdoso), $\mathrm{b}^{*}$ (amarillento-azulado) antes y después del proceso de escaldado, usando un colorímetro Konica Minolta CM-5 (Iluminante D65, ángulo de 10 grados para el observador) mediante reflectancia. Se realizó el cálculo del valor del croma $\left(C^{*}\right)=\left(a^{* 2}+b^{* 2}\right)^{1 / 2}$ y el valor del ángulo de tono $\left(h^{\circ}\right)=180+$ $\tan ^{-1}\left(b^{*} / a^{*}\right)$ para $a^{*}$ negativo y $b^{*}$ positivo (Icier et al., 2006; Padrón et al., 2012).

Clorofila: Se realizó acorde al método de Lichtenthaler y Wellburn (1983). Se maceró 0,5 gr de cada muestra de arveja y se mezcló con acetona al $80 \%$, se recogió el sobrenadante, se repitió el proceso hasta que la muestra se decoloró completamente, se reunieron todos los sobrenadantes y se aforaron con acetona hasta $25 \mathrm{ml}$, se centrifugaron a $3000 \mathrm{rpm}$ durante $5 \mathrm{~min}$ y a $4^{\circ} \mathrm{C}$. Se usó el sobrenadante para realizar las lecturas por espectrofotometría a $663 \mathrm{~nm}$ y $645 \mathrm{~nm}$. Todos los procesos de extracción de clorofila se realizaron a 4 ${ }^{\circ}$ C. Los cálculos de clorofila total (clorofila $a+b$ ) se hicieron mediante la ecuación (3) (Lichtenthaler y Wellburn, 1983; Rodés y Collazo, 2006): 
Clorofila total $\quad \mathrm{Ca}+b\left(\frac{m g}{g}\right)=\left[20.2\left(A_{645}\right)+8.02\left(A_{663}\right)\right] x\left[\frac{v f}{1000 x P}\right]$

Donde $\mathrm{A}_{645}$ y $\mathrm{A}_{663}=$ absorbancia a $645 \mathrm{~nm}$ y $663 \mathrm{~nm}$ respectivamente; vf = volumen al que se aforó la muestra con acetona en $\mathrm{mL} ; 1000$ factor de conversión para obtener unidades de $\mathrm{mg} / \mathrm{g}$ de peso fresco y $\mathrm{P}=$ peso de la muestra en $\mathrm{g}$.

\section{Diseño experimental y análisis de resultados}

Se aplicó un diseño central compuesto $2^{\mathrm{k}}$, con dos factores: temperatura ( 3 niveles: $\left.70,80,90{ }^{\circ} \mathrm{C}\right)$ y tiempo (3 niveles: 1, 2,5 y 4 min) más 2 puntos axiales y 3 puntos centrales. Como variables respuesta se tuvo la actividad residual POD (\%), color, contenido de clorofila y textura (Gökmen et al., 2005). Las variables de respuesta se analizaron con ayuda del programa estadístico StatGraphics Centurion XVI, mediante análisis de varianza, coeficientes de regresión y optimización de respuesta, todo con un nivel de confianza del $95 \%$ (Montgomery y Runger, 2003).

\section{RESULTADOS Y DISCUSIÓN}

\section{Actividad POD en las muestras de arveja sin escaldar}

En las muestras sin escaldar se encontró una actividad enzimática inicial POD $\left(A_{0}\right)$ de $0,0464 \pm 0,0004$ Abs/min para arveja andina y de 0,0371 $\pm 0,0003 \mathrm{Abs} / \mathrm{min}$ para arveja sureña, estos valores permitieron realizar los respectivos cálculos de actividad residual POD en términos de porcentaje para cada muestra escaldada. Las reacciones enzimáticas iniciales se muestran en la Fig. 1 y los resultados de actividad residual para cada tratamiento aplicado se muestran en la Fig. 2.

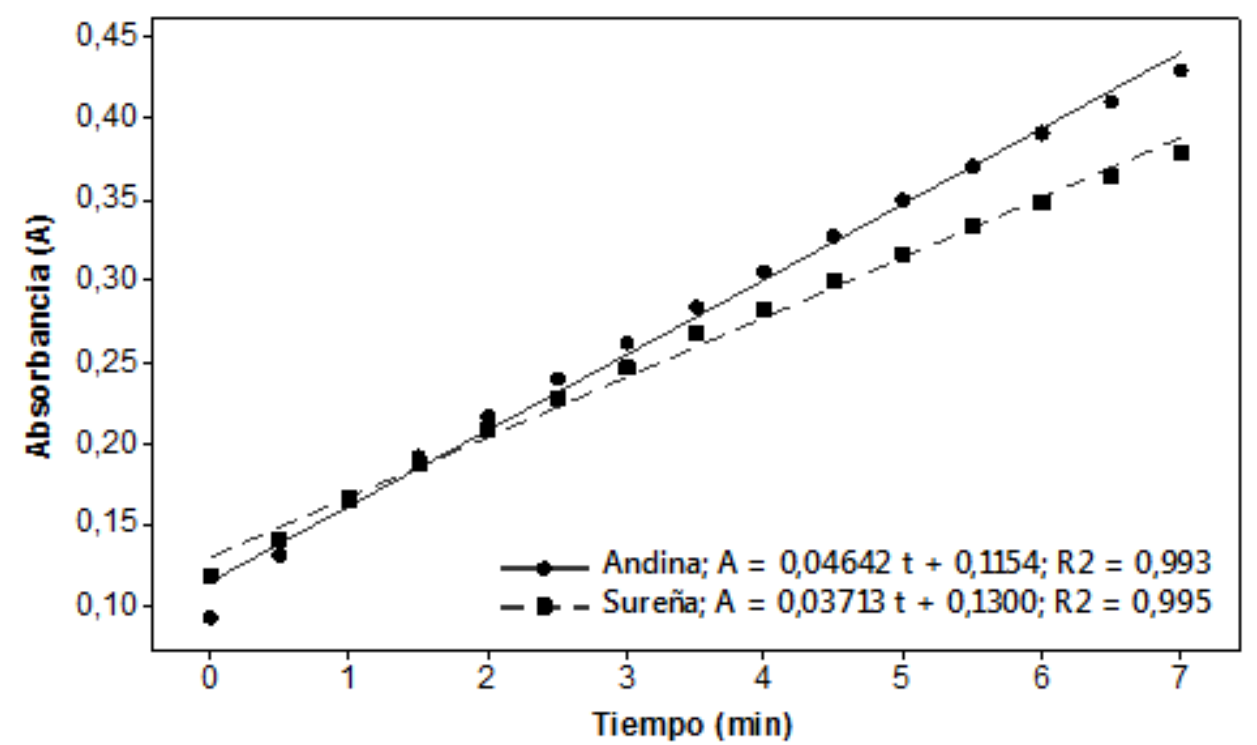

Fig. 1: Actividad enzimática POD en muestras de arveja andina y sureña respectivamente sin escaldar

En la tabla 1 se puede observar que la temperatura, el tiempo y sus respectivos cuadrados (temperaturatemperatura y tiempo-tiempo) ejercen un efecto significativo sobre la reducción en la actividad POD de las dos variedades de arveja (valor $\mathrm{P}<0,05$ ). La interacción temperatura-tiempo solo es significativa en el caso de la variedad andina, ya que para el caso de la variedad sureña esta interacción no tiene efecto sobre la actividad POD. Debido a que en ambos casos los cuadrados de los factores son significativos se puede realizar el proceso de optimización que permitirá definir exactamente cuál es la mejor combinación de temperatura y tiempo que reduce la actividad residual POD hasta valores $\leq 10 \%$.

Este porcentaje de residualidad es suficiente para evitar alteraciones fisicoquímicas en arveja y haba durante el almacenamiento en congelación, lo que a su vez permite inferir que las dos variedades de arveja objeto de este estudio no sufrirían alteraciones fisicoquímicas bajo condiciones de almacenamiento en congelación (Gökmen et al., 2005; Petzold et al., 2014). 


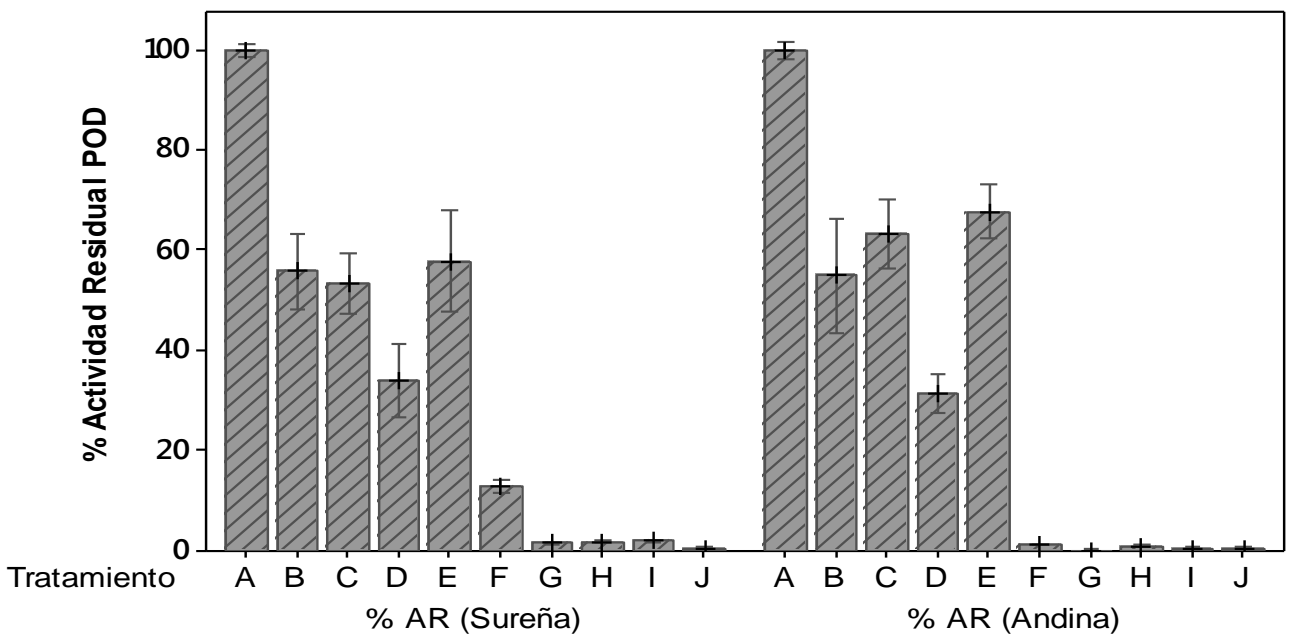

Fig. 2: Actividad residual enzimática POD (\%) en arveja sureña y andina, $\mathrm{A}$, (sin escaldar); $\mathrm{B},\left(66^{\circ} \mathrm{C} x\right.$ $2,5 \mathrm{~min}) ; \mathrm{C},\left(70^{\circ} \mathrm{C} \times 1 \mathrm{~min}\right) ; \mathrm{D},\left(70^{\circ} \mathrm{C} \times 4 \mathrm{~min}\right) ; \mathrm{E},\left(80^{\circ} \mathrm{C} \times 0.37 \mathrm{~min}\right) ; \mathrm{F},\left(80^{\circ} \mathrm{C} \times 2.5 \mathrm{~min}\right) ; \mathrm{G},\left(80^{\circ} \mathrm{C} \times\right.$ $4.6 \mathrm{~min}) ; \mathrm{H},\left(90^{\circ} \mathrm{C} \times 1 \mathrm{~min}\right) ; \mathrm{I},\left(90^{\circ} \mathrm{C} \times 4 \mathrm{~min}\right) ; \mathrm{J},\left(94^{\circ} \mathrm{C} \times 2,5 \mathrm{~min}\right)$.

Tabla 1: ANOVA de análisis de varianza para actividad residual POD en arveja sureña y andina

\begin{tabular}{|c|c|c|c|c|c|c|c|}
\hline Variedad & Fuente & $\begin{array}{l}\text { Suma de } \\
\text { Cuadrados }\end{array}$ & Gl & $\begin{array}{l}\text { Cuadrado } \\
\text { Medio }\end{array}$ & Razón-F & Valor-P & R-cuadrado \\
\hline \multirow{8}{*}{ Sureña } & A:Temperatura & 9768,85 & 1 & 9768,85 & 127,22 & 0,0000 & \multirow{8}{*}{$89,55(\%)$} \\
\hline & B:Tiempo & 3613,01 & 1 & 3613,01 & 47,05 & 0,0000 & \\
\hline & AA & 507,591 & 1 & 507,591 & 6,61 & 0,0174 & \\
\hline & $A B$ & 279,85 & 1 & 279,85 & 3,64 & 0,0694 & \\
\hline & BB & 639,989 & 1 & 639,989 & 8,33 & 0,0086 & \\
\hline & bloques & 8,43805 & 2 & 4,21902 & 0,05 & 0,9467 & \\
\hline & Error total & 1689,36 & 22 & 76,7892 & & & \\
\hline & Total (corr.) & 16166,8 & 29 & & & & \\
\hline \multirow{8}{*}{ Andina } & A:Temperatura & 10859,0 & 1 & 10859,0 & 103,24 & 0,0000 & \multirow{8}{*}{$90,18(\%)$} \\
\hline & B:Tiempo & 6220,83 & 1 & 6220,83 & 59,15 & 0,0000 & \\
\hline & AA & 1819,63 & 1 & 1819,63 & 17,30 & 0,0004 & \\
\hline & $A B$ & 737,587 & 1 & 737,587 & 7,01 & 0,0147 & \\
\hline & BB & 2977,14 & 1 & 2977,14 & 28,31 & 0,0000 & \\
\hline & bloques & 12,4564 & 2 & 6,22821 & 0,06 & 0,9427 & \\
\hline & Error total & 2313,94 & 22 & 105,179 & & & \\
\hline & Total (corr.) & 23575,9 & 29 & & & & \\
\hline
\end{tabular}

\section{Optimización del proceso térmico}

Conforme a los resultados encontrados de actividad residual POD, se realizó el proceso de optimización de respuesta. La fig. 3 muestra la superficie de respuesta para arveja andina y sureña y los valores de temperatura y tiempo optimizados. En la Tabla 2 se puede ver que los mejores tratamientos térmicos estimados para arveja sureña y andina son de $82,32{ }^{\circ} \mathrm{C}$ por $2,58 \mathrm{~min}$ y de $76,98{ }^{\circ} \mathrm{C}$ por $2,56 \mathrm{~min}$ respectivamente. Estos tratamientos térmicos optimizados permiten alcanzar una actividad enzimática residual $\mathrm{POD} \leq 10 \%$. Adicionalmente el diseño estadístico permitió deducir los polinomios (segundo orden) para arveja sureña y andina, mediante los cuales se pueden estimar los valores de actividad residual POD, para los procesos térmicos optimizados (ecuaciones 4 y 5 ).

$$
\begin{aligned}
& \% \text { AR POD }(\text { Sureña })=667.51-12.5564(\mathrm{~T})-49.116(\mathrm{t})+0.06084\left(\mathrm{~T}^{2}\right)+0.3219(\mathrm{~T})(\mathrm{t})+3.036\left(t^{2}\right) \\
& \% \text { AR POD }(\text { Andina })=1080.95-21.8638(\mathrm{~T})-85.288(\mathrm{t})+0.11519\left(\mathrm{~T}^{2}\right)+0.5227(\mathrm{~T})(\mathrm{t})+6.548\left(\mathrm{t}^{2}\right)
\end{aligned}
$$



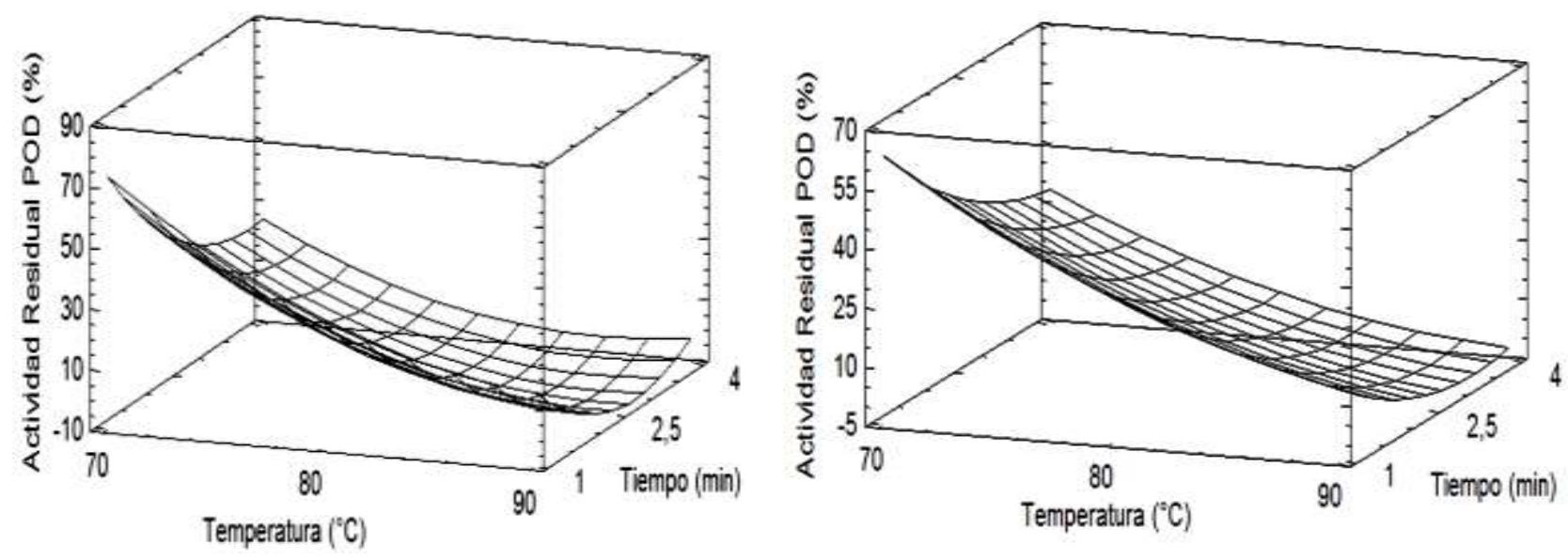

Fig. 3: Respuesta óptima para proceso de escaldado en arveja andina y sureña respectivamente

Tabla 2: Optimización del proceso de escaldado para arveja sureña y andina

\begin{tabular}{|l|l|l|l|l|l|}
\hline Factor & Bajo & Alto & Óptimo Sureña & Óptimo Andina & Unidades \\
\hline Temperatura $(\mathrm{T})$ & 66 & 90 & 82,32 & 76,98 & $\left({ }^{\circ} \mathrm{C}\right)$ \\
\hline Tiempo (t) & 0,37 & 4 & 2,58 & 2,56 & $(\mathrm{~min})$ \\
\hline
\end{tabular}

Considerando las ecuaciones (4) y (5), la actividad residual POD alcanzada para cada tratamiento optimizado es de 8,00 \% para la variedad sureña y de 8,05\% para la variedad andina. Resultados similares encontraron los autores Lin y Brewer (2005), quienes lograron una reducción en la actividad enzimática POD del $97 \%$, además, mostraron que el $3 \%$ restante de la enzima no presenta reactivación durante y después del proceso de congelación en arveja. Adicionalmente, Latorre et al. (2013) afirman que una inactivación del $90 \%$ de la enzima inicial es suficiente para mantener las características fisicoquímicas de los vegetales, las cuales, se pierden normalmente por el efecto de las enzimas. Así mismo, Gökmen et al. (2005) encontraron que no hubo regeneración de la actividad enzimatica POD en arvejas escaldadas a 80 ${ }^{\circ} \mathrm{C}$ durante 2 min y almacenadas a $-18^{\circ} \mathrm{C}$ durante 12 meses. Lo anterior permite inferir que los tratamientos térmicos optimizados para las dos variedades de arveja son idóneos como paso previo al proceso de conservación por congelación, secado o para elaboración de conservas, debido a que logran reducir la actividad enzimática POD a valores $\leq 10 \%$. Además, se garantizaría que no habrá otros cambios provocados por otras enzimas ya que la enzima POD es muy estable al calor en comparación con otras enzimas, infiriendo que las variedades de arveja sureña y andina escaldadas a $82,32{ }^{\circ} \mathrm{C}$ por 2,58 min y a $76,98{ }^{\circ} \mathrm{C}$ por 2,56 min respectivamente, no sufrirían cambios indeseables en la apariencia, color y sabor (Lin y Brewer, 2005; Polata et al., 2009; Petzold et al., 2014).

\section{Textura}

El efecto del proceso de escaldado sobre la firmeza de las muestras se observa en la Fig. 4. En la arveja variedad sureña sin escaldar se obtuvo un valor de firmeza de 36,42 $\pm 4,53 \mathrm{~N}$. De acuerdo con la ecuación (6) para el proceso de escaldado optimizado de $82,32{ }^{\circ} \mathrm{C} \times 2,58 \mathrm{~min}$, se estima que el valor de firmeza será de $24,73 \mathrm{~N}$, resultando en una pérdida de firmeza del $32,09 \%$. Para el caso de arveja andina sin escaldar se encontró un valor de firmeza de 39,49 $\pm 3,87 \mathrm{~N}$. Tomando en cuenta la ecuación (7) y con base en el proceso de escaldado optimizado $76,98{ }^{\circ} \mathrm{C} \times 2,56 \mathrm{~min}$, se estima un valor de firmeza de $24,01 \mathrm{~N}$, provocando una pérdida de firmeza del $39,29 \%$.

Los efectos tanto de la temperatura como del tiempo originan una disminución de la firmeza en las muestras de arveja de las dos variedades durante el proceso de escaldado que se debe, según Latorre et al., (2013) a que la aplicación de tratamientos térmicos (especialmente por inmersión en agua) provocan diversos cambios en las estructuras celulares, principalmente la desorganización de la membrana celular y cambios en los polímeros de la pared celular, causando un ablandamiento de los tejidos que se traduce en una pérdida de firmeza del producto escaldado (Aguilar et al., 1999; Hombre y Castro, 2007; Mendoza y Herrera, 2012).

$$
\begin{aligned}
& \text { Textura sureña }(\mathrm{N})=1.644+1.1139(\mathrm{~T})-3.9804(\mathrm{t})-0.00886\left(\mathrm{~T}^{2}\right)-0.02451(\mathrm{~T})(\mathrm{t})+1.0325\left(\mathrm{t}^{2}\right) \\
& \text { Textura andina }(\mathrm{N})=9.025+0.77493(\mathrm{~T})-3.3001(\mathrm{t})-0.005781\left(\mathrm{~T}^{2}\right)-0.04183(\mathrm{~T})(\mathrm{t})+0.9842\left(\mathrm{t}^{2}\right)
\end{aligned}
$$




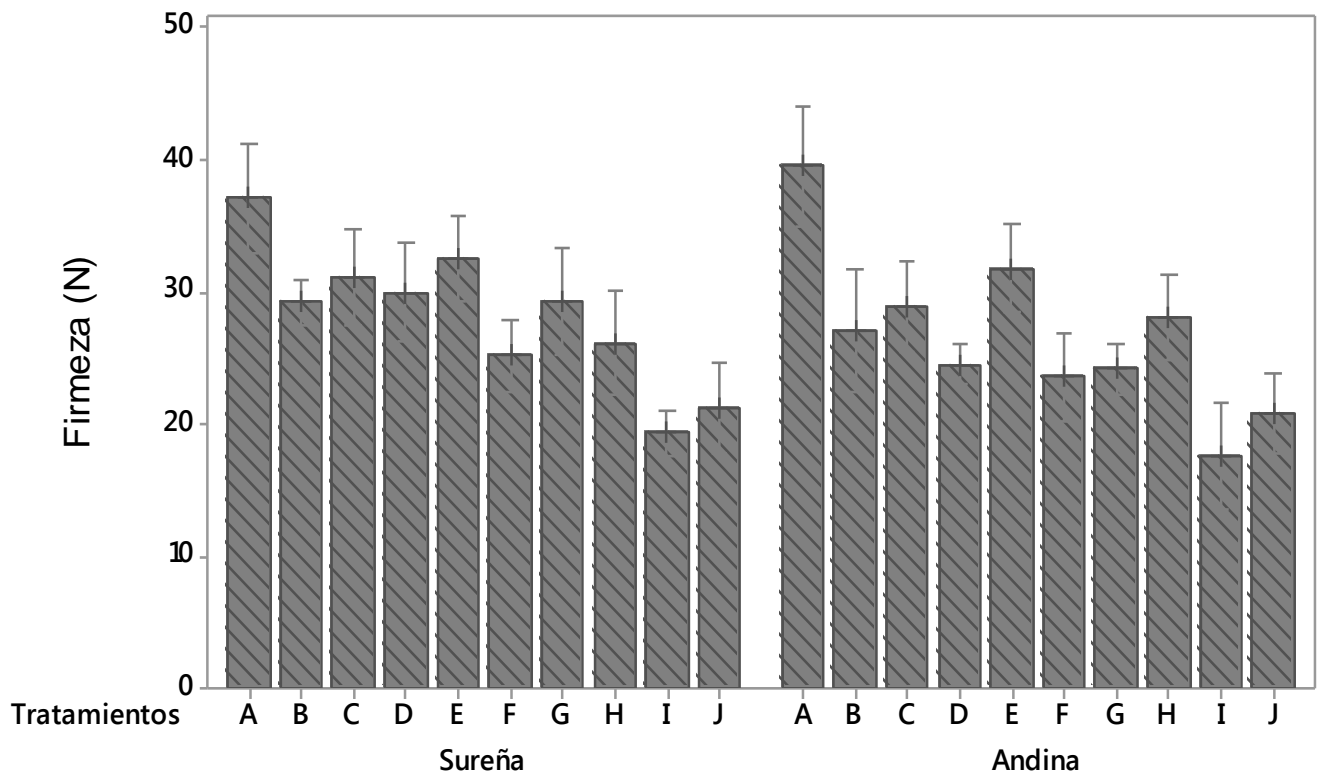

Fig. 4: Efecto del escaldado sobre la textura de las muestras de arveja sureña, $\mathrm{A}$, (sin escaldar); $\mathrm{B},\left(66^{\circ} \mathrm{C} \times 2,5 \mathrm{~min}\right)$; $\mathrm{C},\left(70{ }^{\circ} \mathrm{C} \times 1 \mathrm{~min}\right) ; \mathrm{D},\left(70^{\circ} \mathrm{C} \times 4 \mathrm{~min}\right) ; \mathrm{E},\left(80^{\circ} \mathrm{C} \times 0.37 \mathrm{~min}\right) ; \mathrm{F},\left(80^{\circ} \mathrm{C} \times 2.5 \mathrm{~min}\right) ; \mathrm{G},\left(80^{\circ} \mathrm{C} \times 4.6 \mathrm{~min}\right) ; \mathrm{H},\left(90^{\circ} \mathrm{C} \times 1\right.$ $\min ) ; \mathrm{I},\left(90^{\circ} \mathrm{C} \times 4 \mathrm{~min}\right) ; \mathrm{J},\left(94^{\circ} \mathrm{C} \times 2,5 \mathrm{~min}\right)$.

\section{Clorofila}

La clorofila se ve afectada directamente por el efecto de la temperatura, mientras que el tiempo no ejerce ningún efecto significativo sobre el contenido de clorofila, tal como se muestra en la Fig. 5.

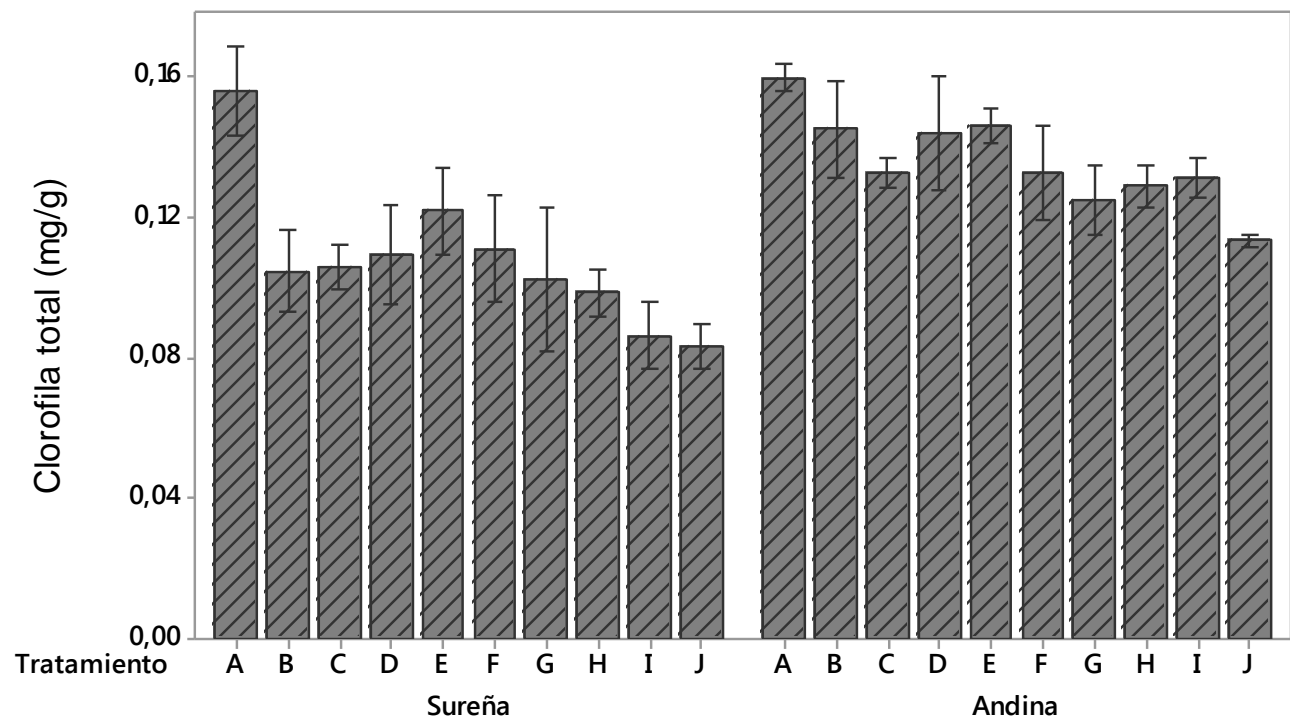

Fig. 5: Contenido de Clorofila en arveja sureña y andina. A, (sin escaldar); $\mathrm{B},\left(66^{\circ} \mathrm{C} \times 2,5 \mathrm{~min}\right) ; \mathrm{C},\left(70{ }^{\circ} \mathrm{C} \times 1 \mathrm{~min}\right) ; \mathrm{D}$, (70 $\left.{ }^{\circ} \mathrm{C} \times 4 \mathrm{~min}\right) ; \mathrm{E},\left(80^{\circ} \mathrm{C} \times 0.37 \mathrm{~min}\right) ; \mathrm{F},\left(80^{\circ} \mathrm{C} \times 2.5 \mathrm{~min}\right) ; \mathrm{G},\left(80^{\circ} \mathrm{C} \times 4.6 \mathrm{~min}\right) ; \mathrm{H},\left(90^{\circ} \mathrm{C} \times 1 \mathrm{~min}\right) ; \mathrm{I},\left(90^{\circ} \mathrm{C} \times 4 \mathrm{~min}\right) ; \mathrm{J}$, $\left(94^{\circ} \mathrm{C} \times 2,5 \mathrm{~min}\right)$.

Como consecuencia del proceso térmico se puede ver que la clorofila se degrada en ambas variedades de arveja por efecto del incremento en la temperatura aplicada. Estudios realizados por Turkmen et al. (2006) y Sánchez et al. (2014) reportaron que la principal desventaja del uso de tratamientos térmicos en vegetales verdes es la conversión de clorofila a feofitina, debido a que la clorofila pierde estabilidad y sufre cambios físico y químicos, obteniéndose a su vez diferentes grados de verdor que están en función de las combinaciones tiempo temperatura aplicadas y del proceso de escaldado utilizado (Tijskens et al., 2001). Adicionalmente, como se puede ver en la Fig. 5, la clorofila se conservó más en la variedad andina y esto también se ve reflejado al estimar el contenido en clorofila para el tratamiento optimizado aplicando las ecuaciones (8) y (9) donde el contenido de clorofila para la variedad sureña sería de $0,124 \mathrm{mg} / \mathrm{g}$ de peso fresco y para la variedad andina sería de $0,133 \mathrm{mg} / \mathrm{g}$ de peso fresco. 


$$
\begin{aligned}
& \text { Clorofila sureña }\left(\frac{\mathrm{mg}}{g}\right)=-0,47+0,015(\mathrm{~T})+0,018(\mathrm{t})-0,000093\left(\mathrm{~T}^{2}\right)-0,00027(\mathrm{~T})(\mathrm{t}) \\
& \text { Clorofila andina }\left(\frac{\mathrm{mg}}{\mathrm{g}}\right)=0,086+0,0018(\mathrm{~T})+0,0058(\mathrm{t})-0,000014\left(\mathrm{~T}^{2}\right)-0,00015(\mathrm{~T})(\mathrm{t})+0,00094\left(\mathrm{t}^{2}\right)
\end{aligned}
$$

\section{Color}

El efecto de la temperatura y del tiempo provocaron cambios en el color que se observan en las Fig. 6. y 7

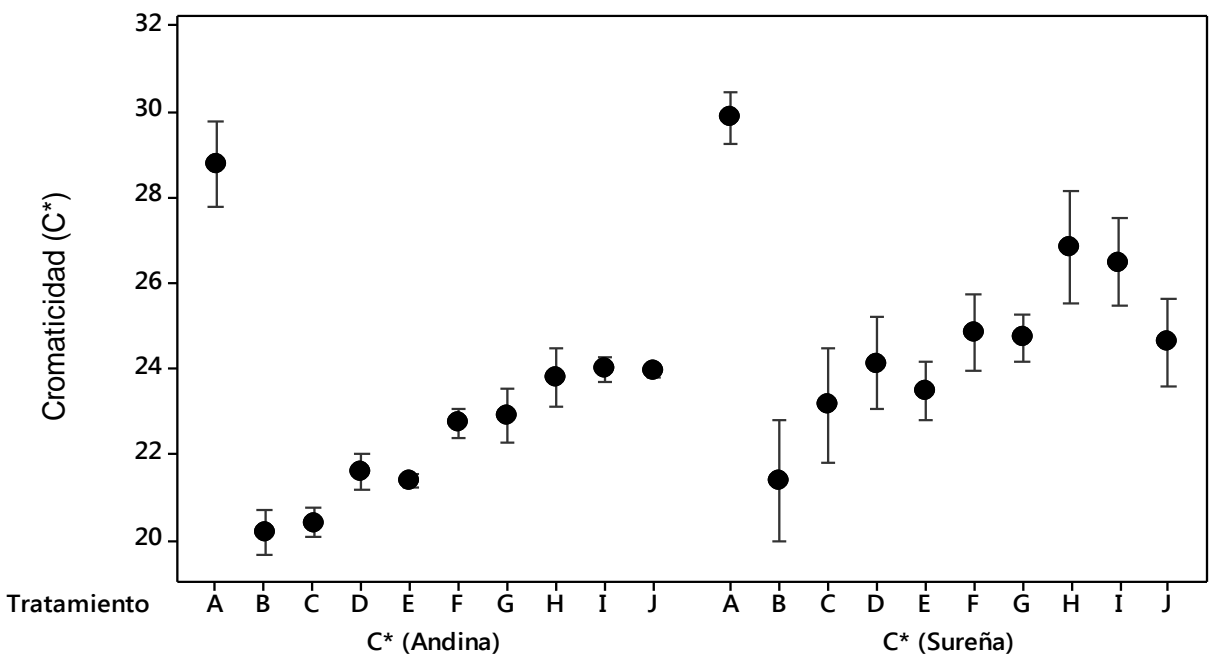

Fig. 6: Variación del color en arveja andina y sureña, $\mathrm{A}$, (sin escaldar); $\mathrm{B},\left(66^{\circ} \mathrm{C} \times 2,5 \mathrm{~min}\right)$; $\mathrm{C},\left(70^{\circ} \mathrm{C} \times 1 \mathrm{~min}\right) ; \mathrm{D},\left(70^{\circ} \mathrm{C} \times 4 \mathrm{~min}\right) ; \mathrm{E},\left(80^{\circ} \mathrm{C} \times 0.37 \mathrm{~min}\right) ; \mathrm{F},\left(80^{\circ} \mathrm{C} \times 2.5 \mathrm{~min}\right) ; \mathrm{G},\left(80^{\circ} \mathrm{C}\right.$ x $4.6 \mathrm{~min}) ; \mathrm{H},\left(90^{\circ} \mathrm{C} \times 1 \mathrm{~min}\right) ; \mathrm{I},\left(90^{\circ} \mathrm{C}\right.$ x $\left.4 \mathrm{~min}\right) ; \mathrm{J},\left(94^{\circ} \mathrm{C} \times 2,5 \mathrm{~min}\right)$.

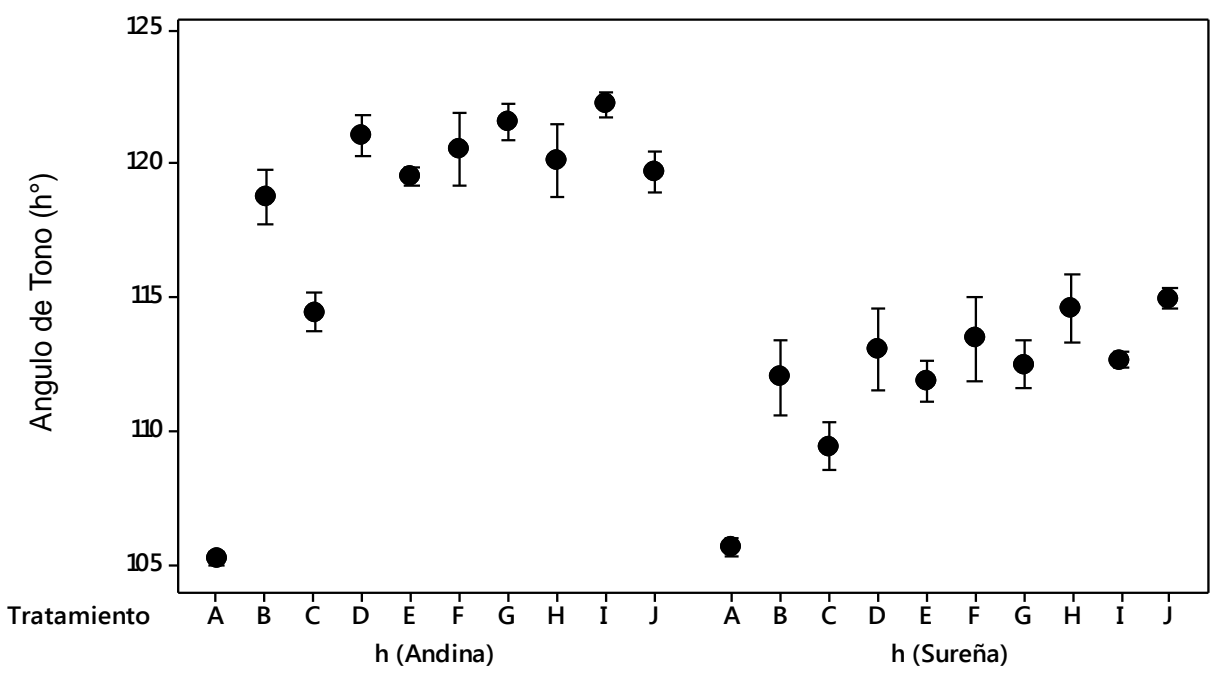

Fig. 7: Variación del color en arveja andina y sureña, $\mathrm{A}$, (sin escaldar); $\mathrm{B},\left(66^{\circ} \mathrm{C} \times 2,5 \mathrm{~min}\right)$; $\mathrm{C},\left(70^{\circ} \mathrm{C} \times 1 \mathrm{~min}\right) ; \mathrm{D},\left(70^{\circ} \mathrm{C} \times 4 \mathrm{~min}\right) ; \mathrm{E},\left(80^{\circ} \mathrm{C} \times 0.37 \mathrm{~min}\right) ; \mathrm{F},\left(80^{\circ} \mathrm{C} \times 2.5 \mathrm{~min}\right) ; \mathrm{G},\left(80^{\circ} \mathrm{C}\right.$ x $4.6 \mathrm{~min}) ; \mathrm{H},\left(90^{\circ} \mathrm{C} \times 1 \mathrm{~min}\right) ; \mathrm{I},\left(90^{\circ} \mathrm{C} \times 4 \mathrm{~min}\right) ; \mathrm{J},\left(94^{\circ} \mathrm{C} \times 2,5 \mathrm{~min}\right)$.

Los resultados mostrados en la Fig. 7, fueron similares y tuvieron el mismo comportamiento que se presentó en espárragos, judías verdes y calabacín escaldados por inmersión en agua hirviendo a 100 ㄷ (Mazzeo et al., 2015), es decir, el grado de verdor aumenta cuando se aplica un tratamiento térmico. En arveja andina este cambio en el ángulo de tono (grado de verdor) fue más representativo que en arveja sureña, ya que en muestras de arveja andina sin escaldar el ángulo de tono estuvo alrededor de los 106ํy después de aplicar el tratamiento térmico estuvo en un rango desde los $113^{\circ}$ hasta los $123^{\circ}$, mientras que en muestras de arveja sureña sin escaldar el ángulo de tono estuvo alrededor de los $107^{\circ}$ y después de aplicar el proceso de escaldado estuvo en un rango desde los 109 hasta los $114^{\circ}$. Este cambio de color presentado en las dos variedades de arveja también se puede visualizar en la variación que se presenta para el valor de cromaticidad (Fig. 6) ya que en muestras sin escaldar el color es más puro y en muestras escaldadas la pureza del color disminuye, no obstante, a medida que el tratamiento térmico aplicado es más severo la 
pureza aumenta, pero no alcanza la cromaticidad de las muestras sin escaldar. Estos cambios de color se presentan principalmente por la remoción del aire atrapado entre los tejidos de los materiales vegetales por el agua usada durante el proceso de escaldado (Mazzeo et al., 2015), además, tomando en cuenta que el color verde de la arveja lo otorga la clorofila, el cambio de color se presenta por la degradación de la clorofila a feofitina y otros productos de degradación (Sánchez et al., 2014; Turkmen et al., 2006), lo que permite inferir que la variación del color está dada por la severidad del tratamiento térmico que afecta principalmente a la clorofila presente en las dos variedades de arveja (Tijskens et al., 200; Icier et al., 2006; Padrón et al., 2012).

\section{CONCLUSIONES}

A partir de los resultados obtenidos, se puede concluir: 1) Mediante proceso de optimización estadístico se estimó que la actividad enzimática de peroxidasa se reducirá a valores $\leq 10 \%$, y como resultado, el proceso permitió obtener tratamientos térmicos no tan severos, debido a sus tiempos cortos de exposición al calor $\left(76,98^{\circ} \mathrm{C}\right.$ x 2,56 min y de $82,32{ }^{\circ} \mathrm{C}$ x 2,58 min para arveja andina y sureña respectivamente) lo que permitirá conservar mejor las propiedades fisicoquímicas del producto escaldado; 2) El proceso de escaldado por inmersión en agua provocó disminución de la firmeza en las dos variedades de arveja. En este sentido y tomando en cuenta la optimización de los tratamientos térmicos, se espera que la variedad sureña sea la que más soporte la pérdida de firmeza en comparación con la variedad andina, ya que los valores de pérdida de firmeza son de 32,09 \% y de 39,29 \% respectivamente; 3) Como consecuencia del proceso térmico la clorofila se degradó en ambas variedades de arveja, sin embargo, la clorofila se conservó mejor en la variedad andina, la cual presentó mayor concentración de este pigmento en comparación con la variedad sureña; 4) El proceso de escaldado permitió resaltar la tonalidad del color verde de la arveja obteniendo un verde más oscuro con respecto a las muestras sin escaldar, no obstante, la cromaticidad de las muestras escaldadas se ve afectada en comparación con las muestras sin escaldar.

\section{REFERENCIAS}

Agronet, Ministerio de Agricultura y Desarrollo Rural. República de Colombia (en línea), http://www.agronet.gov.co/www/htm3b/ReportesAjax/parametros/reporte25_2011.aspx?cod=25. Acceso: Enero de 2015 (2013)

Aguilar, C. N., M. Reyes, H. De la Garza y J. Contreras-Esquivel, Aspecto bioquímicos de la relación entre el escaldado TB-TL y la textura de vegetales procesados, http://www.redalyc.org/articulo.oa?id=47543104; ISSN: 1870-249X, Revista de la Sociedad Química de México: 43 (2), 54-62 (1999)

Anthon, G. E. y D. M. Barrett, "Kinetic parameters for the thermal inactivation of quality-related enzymes in carrots and potatoes", doi: 10.1021/jf011698i, J. Agric. Food Chem., 50 (14), 4119-4125 (2002)

Hombre R. y E. Castro, Parámetros mecánicos y textura de los Alimentos, Universidad de Chile, Santiago, Chile, Disponible en: http://www.repositorio.uchile.cl/handle/2250/121381, (2007)

Featherstone S., A Complete Course in Canning and Related Processes, 14a edición, vol. 2, 43-58. Woodhead Publishing, Oxford (2015)

Gökmen, V., K. Savaş Bahçeci, A. Serpen, y J. Acar, Study of lipoxygenase and peroxidase as blanching indicator enzymes in peas: Change of enzyme activity, ascorbic acid and chlorophylls during frozen storage, doi:10.1016/j.Iwt.2004.06.018, LWT - Food Science and Technology, 38 (8), 903-908 (2005)

Huerta, V. M., R. Díaz y A. Hernández, Efecto de los tratamientos sobre la textura de la jícama elaborada por procesamiento mínimo, Ciencia y Tecnología de Alimentos: 18 (2), 54-58 (2008)

Icier, F., H. Yildiz y T. Baysal, "Peroxidase inactivation and colour changes during ohmic blanching of pea puree", doi: 10.1016/j.jfoodeng.2005.03.032, Journal of Food Engineering, 74(3), 424-429 (2006)

Lichtenthaler, H. y A. Wellburn, "Determinations of total carotenoids and chlorophylls $b$ of leaf extracts in different solvents", doi:10.1042/bst0110591, Biochemical Society Transactions, 11 (5), 591-592 (1983)

Latorre, M. E., M. F. de Escalada Plá, A. M. Rojas, L. N. Gerschenson, "Blanching of red beet (Beta vulgaris L. var. conditiva) root, Effect of hot water or microwave radiation on cell wall characteristics", doi:10.1016/j.Iwt.2012.06.004, LWT - Food Science and Technology, 50 (1), 193-203 (2013) 
Latorre, V. L., C. A. Pantoja, D. Mejía, M. Osorio y A. Hurtado, "Evaluación de tratamientos térmicos para inactivación de enzimas en jugo de fique (Furcraea gigantea Vent.)", http://www.scielo.org.co; ISSN 1692-3561, Biotecnología en el Sector Agropecuario y Agroindustrial: 11 (1), 113 - 122 (2013)

Lin, S. y M. S. Brewer, "Effects of blanching method on the quality characteristics of frozen peas", doi: 10.1111/j.1745-4557.2005.00038.x, Journal of Food Quality, 28 (4), 350-360 (2005)

Macías, S. M., C. Mantell, M. Rodríguez, E. Martínez de la Ossa, L. Lubían, y O. Montero, "Supercritical fluid extraction of carotenoids and chlorophyll a from Synechococcus sp", doi:10.1016/j.supflu.2006.03.008, Journal of Supercritical Fluids, 39, 323-329 (2007)

Mazzeo. T., M. Paciulli, E. Chiavaro, A. Visconti, V. Fogliano, T. Ganino, N. Pellegrini, "Impact of the industrial freezing process on selected vegetables -Part II. Colour and bioactive compounds", doi:10.1016/j.foodres.2015.05.036, Food Res. Int., 75, 89-97 (2015)

Mendoza, R, y A. O. Herrera, "Cinética de Inactivación de la Enzima Peroxidasa, Color y Textura en Papa Criolla (Solanum tuberosum Grupo phureja) sometida a tres Condiciones de Escaldado", doi:10.4067/S0718-07642012000400009, Inf. Tecnol. 23 (4), 73-82 (2012)

Montgomery, D. C. y G. C. Runger, Applied Statistics and Probability for Engineers, $3^{\text {a }}$ Edition, I. John Wiley \& Sons, Ed. New York, United States of America (2003)

Padrón Pereira, C. A., G. M. Padrón León, A. I. Montes Hernández, y R. A. Oropeza González, "Determinación del color en epicarpio de tomates (Lycopersicum esculentum Mill.) con sistema de visión computarizada durante la maduración", http://www.mag.go.cr/rev_agr/index.html; ISSN:0377-9424, Agronomía Costarricense, 36(1), 97-111 (2012)

Petzold G., C. Marisa y J. Moreno, "Influence of blanching, freezing and frozen storage on physicochemical properties of broad beans (Vicia faba L)", doi:10.1016/j.jirefrig.2013.05.007, International journal of refrigeration, 40, 429-434 (2014)

Pinchao, Y. A., O. Osorio y D. Mejía, "Inactivación Térmica de Pectinmetilesterasa en Jugo de Uchuva (Physalis peruviana L.)", doi: 10.4067/S0718-07642014000500009, Inf. Tecnol. 25(5), 55-64 (2014)

Polata, H., A. Wilinska, J. Bryjak, M. Polakovic, "Thermal inactivation kinetics of vegetable peroxidases", doi:10.1016/j.jfoodeng.2008.09.017, Journal of Food Engineering, 91 (3), 387-391 (2009)

Rodés, G. R. y O. M. Collazo, Manual de prácticas de fotosíntesis, $1^{\text {ra }}$ edición, Las prensas de ciencias, México (2006)

Sánchez, C., A. B. Baranda, y I. M. De Marañón, "The effect of High Pressure and High Temperature processing on carotenoids and chlorophylls content in some vegetables", doi:10.1016/j.foodchem.2014.04.041, Food Chemistry, 163, 37-45 (2014)

Tijskens, L.M.M., E.P.H.M. Schijvens, y E.S.A. Biekman, "Modelling the change in colour of broccoli and green beans during blanching", doi:10.1016/S1466-8564(01)00045-5, Innovative Food Science and Emerging Technologies, 2, 303-313 (2001)

Tiwari, B. K., K. Muthukumarappan, C. P. O'donnell y P. J. Cullen, "Inactivation Kinetics of pectin methylesterase and cloud retention in sonicated orange juice", doi:10.1016/j.ifset.2008.11.006, Innovative Food Science \& Emerging Technologies, 10 (2), 166-171 (2009)

Torres, R., E. J. Montes, O. A. Pérez, R. D. Andrade, "Relación del Color y del Estado de Madurez con las Propiedades Fisicoquímicas de Frutas Tropicales", doi: 10.4067/S0718-07642013000300007, Inf. Tecnol. 24 (3), 51-56 (2013)

Turkmen, N. P., F. Sari y V. Y. Sedat, "Effects of cooking methods on chlorophylls, pheophytins and colour of selected green vegetables", doi:10.1111/j.1365-2621.2005.01061.x, International Journal of Food Science and Technology, 41, 281-288 (2006) 